\title{
Green Synthesis of AgNPs Using Lignocellulose Nanofibrils as a Reducing and Supporting Agent
}

\author{
Jun-Kyu Han, ${ }^{\mathrm{a}}$ Alle Madhusudhan, ${ }^{\mathrm{b}}$ Rajkumar Bandi, ${ }^{\mathrm{b}}$ Chan-Woo Park, ${ }^{\mathrm{d}}$ Jin-Chul Kim, ${ }^{\mathrm{c}}$ \\ Yong-Kyu Lee, ${ }^{\text {a }}$ Seung-Hwan Lee, ${ }^{\mathrm{d}, *}$ and Jong-Myoung Won ${ }^{\mathrm{a}, *}$
}

\begin{abstract}
A novel green approach was developed for producing silver nanoparticles (AgNPs) using lignocellulose nanofibrils (LCNF). This method does not require any additional reducing agent, and the LCNF itself serves both as a reducing agent and a supporting material. A simple autoclave procedure was employed for the synthesis. The synthetic conditions such as concentrations of reactants and reaction time were optimized. Also, the effect of lignin content in LCNF on the formation of AgNPs was evaluated. Three types of cellulose nanofibrils, i.e., HCNF (0\% lignin), LCNF-5 (5\% lignin), and LCNF-18 (18\% lignin), were employed for the preparation of AgNPs. Three types of AgNPs were obtained and thoroughly characterized using UV-vis, Fouriertransform Infrared spectroscopy (FT-IR), transmission electron microscopy (TEM), X-ray diffraction (XRD), and X-ray photoelectron spectroscopy (XPS). The results suggest that LCNF can be employed as a green source for the reduction and effective stabilization of AgNPs, but an increased content of lignin can have an adverse effect on the yield of AgNPs. However, the presence of lignin greatly influenced the particle size. Therefore, LCNF with small amounts of lignin (5\%) is best for producing AgNPs.
\end{abstract}

Keywords: Lignocellulose nanofibril (LCNF); Green synthesis; Silver nanoparticles; Composition of $L C N F$

Contact information: a: Department of Paper Science and Engineering, College of Forest and Environmental Sciences, Kangwon National University, Chuncheon, 24341, Republic of Korea; $b$ : Institute of Forest Science, Kangwon National University, Chuncheon, 24341, Republic of Korea; c: Department of Medical Biomaterials Engineering, College of Biomedical Science and Institute of Bioscience and Biotechnology, Kangwon National University, Chuncheon 24341, Republic of Korea; d: Department of Forest Biomaterials Engineering, College of Biomedical Science and Institute of Bioscience and Biotechnology, Kangwon National University, Chuncheon 24341 Republic of Korea;

*Corresponding author: lshyhk@kangwon.ac.kr,wjm@kangwon.ac.kr

\section{INTRODUCTION}

Nanostructured materials find a wide range of applications in the fields of optoelectronics, photonics, catalysis, and medicine (Khan et al. 2017; Madhusudhan et al. 2019). Noble metal nanoparticles, especially silver, have gained a large amount of attention on account of their optical, electrochemical properties, and strong antimicrobial effects (Abou El-Nour et al. 2010; Natsuki 2015). Abundant research reports have revealed the outstanding antimicrobial, anti-platelet, anti-inflammatory, anticancer and antiviral properties of silver nanoparticles (AgNPs) (Prabhu and Poulose 2012; Ovais et al. 2016; Pugazhendhi et al. 2018). Besides their antimicrobial nature, AgNPs are also well known for their antiplasmodial, larvicidal, leishmanicidal, scolicidal and pupicidal activity (Suman et al. 2013; Arokiyaraj et al. 2015; Saratale et al. 2017). These 
fascinating properties have driven researchers to explore several synthetic routes for the production of AgNPs.

Traditionally, physical and chemical methods are widely employed for producing AgNPs. However, the physical process requires a high energy input, and the chemical method is expensive and uses harmful ingredients (Rafique et al. 2017). Alternative methods that ensure sustainability have been developed in recent years. Considerable efforts have been made towards the eco-friendly synthesis with the help of green chemistry principles (Rajoriya et al. 2017). For instance, Saravanan et al. 2018 reported biological synthesis of AgNPs using Bacillus bervis (NCIM 2533) and studied the antibacterial properties against multi-drug resistant pathogens such as Salmonella typhi and Staphylococcus aureus. Oves et al. (2018) used root hair extract of Phoenix dactylifera for the production of AgNPs and studied their antimicrobial and anticancer activity. Similarly, Habibipour et al. 2019 prepared AgNPs using pomegranate peel extract and evaluated their anti-biofilm formation activity against Pseudomonas aeruginosa. The development of biogenic methods generally involves the use of microorganisms and plant extracts (Narayanan and Sakthivel 2010; Ahmed et al. 2016). In comparison to plant extracts, microorganisms present notable disadvantages, such as the time required to obtain them (more than $24 \mathrm{~h}$ ), health risk, and complications involved in maintaining cultures. Thus, the use of plant extracts in the production of metal nanoparticles is of high interest.

Cellulose is the most abundant fibrous organic biopolymer in nature; it consists of $\beta$-1,4-linked anhydro-D-glucose units. It is the chief structural component of plants and is also found in marine animals, algae, fungi, bacteria, invertebrates, and amoeba (Van Rie and Thielemans 2017). Cellulose is renewable, lightweight, and biodegradable. It is present in the form of microfibrils surrounded by hemicellulose and lignin in the cell walls of the plants. Cellulose nanofibril (CNF, also known as nanofibrillated cellulose) is a material composed of nanosized cellulose fibrils with average widths of 5 to $20 \mathrm{~nm}$ and has a wide range of lengths (Park et al. 2017a). CNF can be obtained by mechanical treatment of wood with strong shear forces such as a grinder, fluidizer, and homogenizer. On the basis of chemical composition, CNF can be categorized as lignocellulose nanofibrils (LCNF) that contain both lignin and hemicellulose, holocellulose nanofibrils (HCNF) that contain only hemicellulose and no lignin, and pure cellulose nanofibrils (PCNF) that comprise only the cellulose component (Park et al. 2017b).

AgNPs are well known for their antimicrobial properties, but in the colloidal solution state the AgNPs tend to aggregate in physiological solutions (Lee et al. 2007; Li and Lenhart 2012). This aggregation limits the applicability of AgNPs, as smaller AgNPs colloids possess superior antibacterial activity compared to larger AgNPs aggregates (Wang et al. 2014). Various polymers were employed as stabilizing agents to limit the aggregation of AgNPs in physiological media (Wang et al. 2014). It is logical to expect that CNF will best serve this purpose due to its large number of hydroxyl groups. There are some reports on the production of CNF supported AgNPs, but they suffer from drawbacks including the required use of toxic chemicals and long reaction times. For instance, one study prepared a cellulose fiber/AgNPs composite using alkalis $\left(\mathrm{NH}_{3}\right.$, $\mathrm{NaOH}$, and $\mathrm{Na}_{2} \mathrm{CO}_{3}$ ) as activation agents (Xu et al. 2016). A study in 2018 prepared gold, silver, and nickel nanoparticles anchored in cellulose nanofibers, where $\mathrm{NaBH}_{4}$ was used for reducing metal ions (Gopiraman et al. 2018). Another study prepared hydrogel, aerogel, and the film of CNF functionalized with AgNPs (Dong et al. 2013). Here, the $\mathrm{Ag}^{+}$ions are spontaneously reduced to AgNPs, but it required a long reaction time of 5 
days. Thus, developing a new method to prepare AgNPs functionalized CNF without the use of toxic chemicals and long reaction time is of great need.

In an attempt to address these issues, the authors have explored the possible utilization of LCNF as reducing and stabilizing agent for producing AgNPs. Novelty of the present work lies in the utilization of LCNF with different chemical composition as reducing and stabilizing agent. A simple autoclave procedure was employed for the synthesis and process parameters, such as the concentration of reactants (LCNF and $\mathrm{AgNO}_{3}$ ) and reaction time were optimized. Furthermore, the effect of lignin content of LCNF on the formation of AgNPs was evaluated. For this purpose, three types of cellulose nanofibrils, i.e., HCNF (0\% lignin), LCNF-5 (5\% lignin), and LCNF-18 (18\% lignin), were employed for the preparation of AgNPs. Three types of AgNPs were thoroughly characterized using UV-vis, Fourier-transform infrared spectroscopy (FTIR), transmission electron microscopy (TEM), X-ray diffraction (XRD), and X-ray photoelectron spectroscopy (XPS).

\section{EXPERIMENTAL}

\section{Materials}

A wood sample for the preparation of LCNF was obtained from a Korean red pine (Pinus densiflora) tree present in the Kangwon National University's Experimental Forest. Benzene, ethanol, sodium chlorite, acetic acid, and silver nitrate were procured from Daejung Chemical \& Materials Co. (Siheung, Republic of Korea). Other chemicals were obtained from commercial sources and used without further purification. Deionized water was used throughout the experiment.

\section{Preparation of LCNF}

Wood powder was prepared by pulverizing pine chips, and alcohol-benzene (1 to 2 , volume per volume) extraction was performed to remove the extractives. The extractives content of the sample was measured according to TAPPI T204 cm-97 (2002). The extracted wood powder was delignified with $\mathrm{NaClO}_{2}$ and $\mathrm{CH}_{3} \mathrm{COOH}$ (Cao et al. 2014). The Klason lignin was determined by TAPPI standard method T222 om-06 (2006); the hydrolysis filtrate was further used for the determination of acid-soluble lignin content according to Hyman et al. (2016). The contents of cellulose, hemicellulose, lignin, and extractives of Pinus densiflora wood sample were 35.6\%, 21.4\%, 38\%, and $5 \%$ respectively. The lignin content of extracted wood powder was adjusted to $5 \%$ and $18 \%$ by a delignification reaction. The wood powder partially delignified was subjected to mechanical pre-treatment using a grinder (Super Masscolloider, Masuko Sangyo Co., Ltd., Tokyo, Japan), and then the LCNF was prepared by a high pressure homogenizer (MN400BF, Micronox, Seongnam-si, Korea). The prepared CNF was diluted to a concentration of $0.001 \%$ and filtered at a vacuum of 0.8 bar using a membrane filter (pore size: $0.2 \mu \mathrm{m}$, diameter: $47 \mathrm{~mm}$ ). Solvent substitution was performed four times (15 min each) with tert-butanol $\left(\mathrm{C}_{4} \mathrm{H}_{10} \mathrm{O}\right.$, Daejung Chemicals \& Metals, Siheung-si, Korea) and then freeze dried for $72 \mathrm{~h}$.

\section{Synthesis of AgNPs}

For the synthesis of AgNPs, $1 \mathrm{~mL}$ of $\mathrm{AgNO}_{3}$ solution was added to the $3 \mathrm{~mL}$ of LCNF in a $20 \mathrm{~mL}$ vial; the reaction mixture was shaken well and treated in an autoclave 
at $120{ }^{\circ} \mathrm{C}$. Optimal reaction conditions for the formation of AgNPs were identified by systematically varying the parameters such as reaction time, concentration of LCNF (0.005 wt \% to $1.25 \mathrm{wt} \%$ ), and concentration of $\mathrm{AgNO}_{3}$ (from $0.1 \mathrm{mM}$ to $1.25 \mathrm{mM}$ ).

\section{Characterization}

A picture was taken with an ultra-high-resolution scanning electron microscope (Hitachi S-4800, Tokyo Japan) to observe the morphology of the LCNF prepared for each condition. UV-vis absorption spectra were recorded on a UV-vis spectrophotometer (Jenway, Stone, UK) within the range of 300 to $700 \mathrm{~nm}$. The morphology and size of the synthesized AgNPs were studied using TEM (LEO-912AB OMEGA, LEO, Jena, Germany) at KBSI (Chuncheon, Korea). An X-ray diffraction apparatus ( $\mathrm{Cu}$ target; DMAX 2100V, Rigaku, Tokyo, Japan) operating at $40 \mathrm{kV}$ and $40 \mathrm{~mA}$ was used to evaluate the crystalline nature of AgNPs. The mechanism of formation of AgNPs was confirmed by using FTIR spectra (FT-3000-Excalibur, Varian Inc., Palo Alto, USA) with a scanning range of 400 to $4000 \mathrm{~cm}^{-1}$. XPS data for synthesized AgNPs were obtained with an X-ray photoelectron spectrometer (Omicron Nano Technology, Klaus-Weiler, Austria) with a 128-channel collector.

\section{RESULTS AND DISCUSSION}

\section{CNF SEM Image}

Figure 1 shows the SEM images of HCNF, LCNF-5\%, and LCNF-18\% under different magnifications.

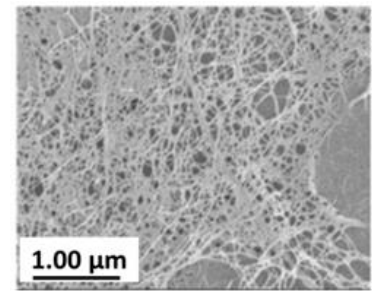

$\operatorname{HCNF}(\mathbf{x 3 0 , 0 0 0 )}$

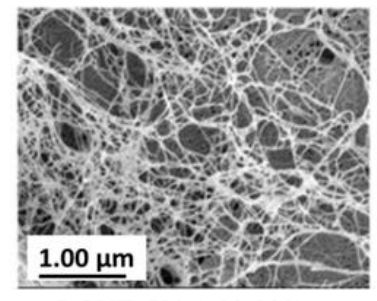

LCNF-5\% (x30,000)

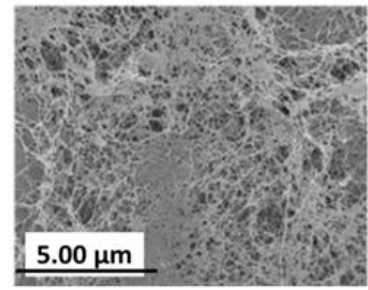

LCNF-18\% (x10,000)

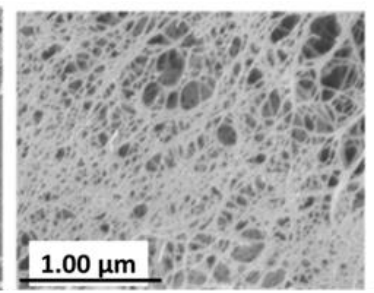

HCNF (x50,000)

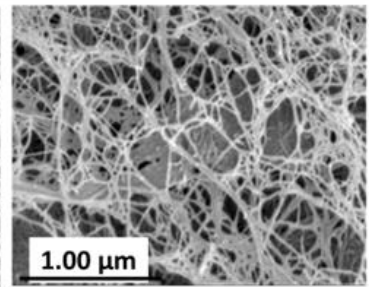

LCNF-5\% (x50,000)

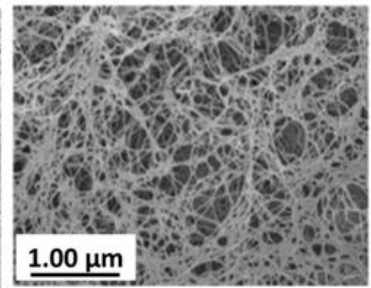

LCNF-18\% (x30,000)

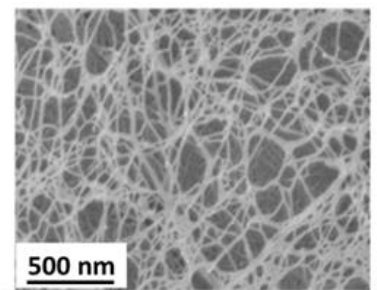

HCNF (x60,000)

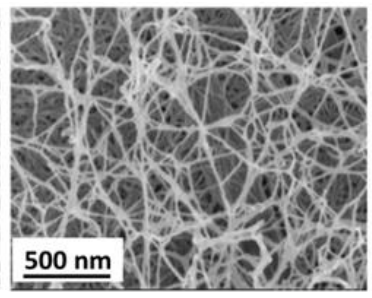

LCNF-5\% (x60,000)

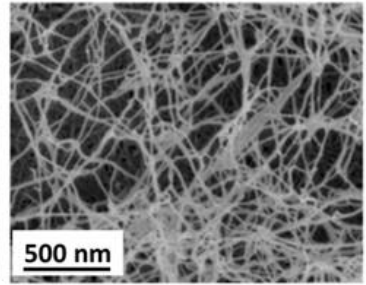

LCNF-18\% (x60,000)

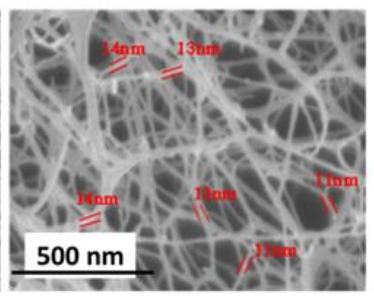

$\operatorname{HCNF}(x 100,000)$

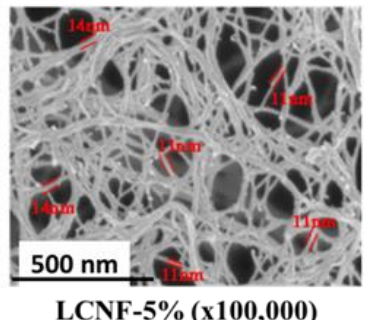

LCNF-5\% (x100,000)

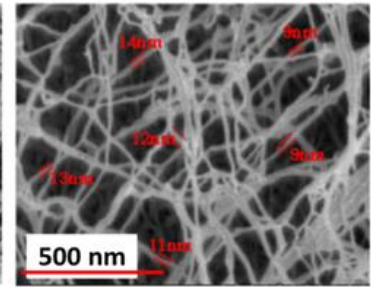

LCNF-18\% (x100,000)

Fig. 1. SEM images of HCNF, LCNF-5\%, and LCNF-18\% 
The observed fibrillar structure confirms the successful formation of nanofibrils. The fibril diameters of all the samples prepared using the homogenizer were uniform and in the size range of $11 \mathrm{~nm}$ to $14 \mathrm{~nm}$. Interestingly, no large difference in fibrillation characteristics was observed between HCNF (0\% lignin), LCNF-5\%, and LCNF-18\% containing lignin.
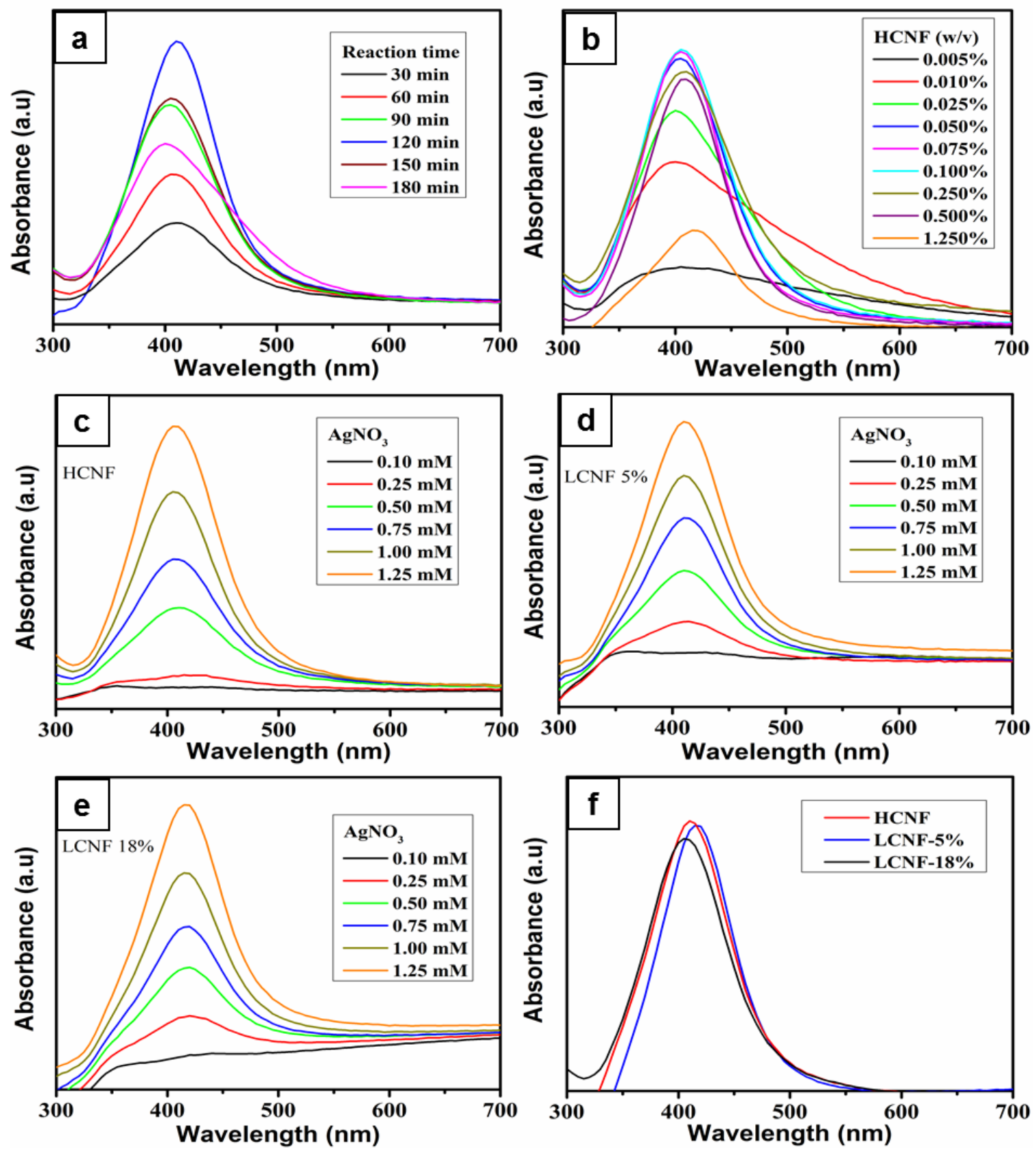

Fig. 2. UV-vis absorption spectra of AgNPs synthesized at different conditions. (a) Different time intervals (b) Different concentration intervals of HCNF (c) HCNF/AgNPs synthesized at different $\mathrm{AgNO}_{3}$ concentration intervals (d) $\mathrm{LCNF}(5 \%) / \mathrm{AgNPs}$ synthesized at different $\mathrm{AgNO}_{3}$ concentration intervals, (e) LCNF(18\%)/AgNPs synthesized at different $\mathrm{AgNO}_{3}$ concentration intervals, (f) Comparison of SPR peak of AgNPs produced with different CNFs under optimal reaction conditions. 


\section{UV-Vis Spectroscopic Investigation of Silver Nanoparticles Formation}

The synthetic conditions (reaction time and dosage of nanofibrils) were optimized to produce AgNPs using HCNF (0\% lignin) as a reducing and stabilizing agent (Fig. 2a,b). Next, under the optimal conditions the formation of AgNPs using LCNF-5\% and LCNF-18\% were studied to assess the effect of lignin content on AgNPs production (Fig 2c,d\&e). However, comparison among the HCNF, LCNF-5\%, and LCNF-18\% (Fig. 2f) revealed that the AgNPs formation was high (higher SPR peak intensity) for HCNF, followed by LCNF-5\% and LCNF-18\%. These results indicate the lesser formation of AgNPs when using LCNF with increased content of lignin. The SPR peak position of three samples is observed at different wavelengths indicating the difference in the particle size distribution (which is further analyzed using TEM).

\section{TEM Analysis}

Figure 3 shows the TEM images of HCNF, LCNF-5\%, and CNF-18\% alone as well as the TEM images of AgNPs synthesized using HCNF, LCNF-5\%, and CNF-18\%.

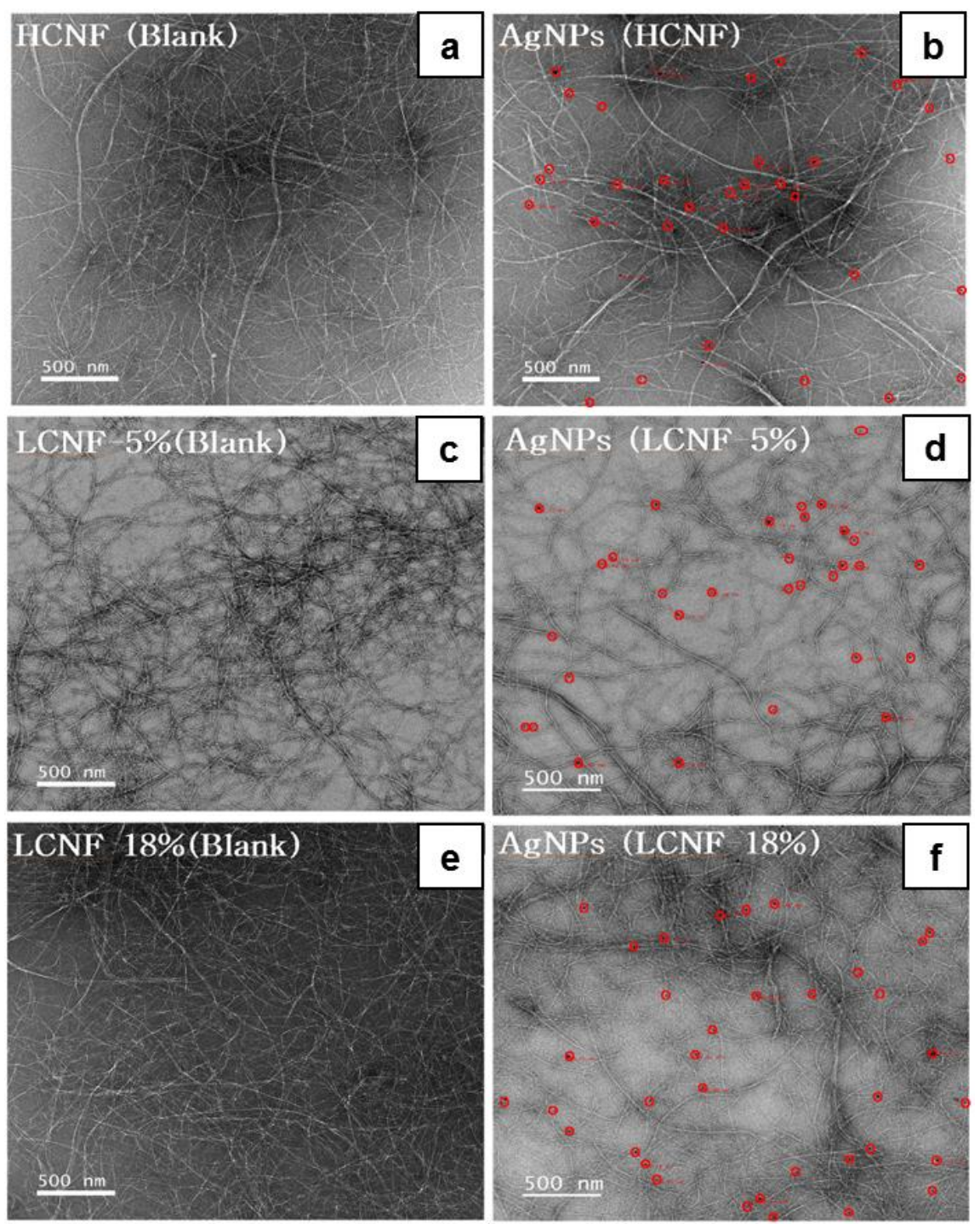

Fig. 3. Low magnification TEM images of (a) HCNF-Blank, (b) AgNPs/HCNF, (c) LCNF 5\%Blank, (d) AgNPs/LCNF-5\%, (e) HCNF 18\%-Blank, and (f) AgNPs/LCNF18\% 
The CNF were clearly present in their nanofibrillar structure. The TEM images of AgNPs clearly shows that the AgNPs had spherical morphology and were well distributed on the nano fibers without any aggregation. High magnification TEM images of AgNPs and their corresponding particle size distribution histograms are shown in Fig. 4. The average particle size of AgNPs synthesized when HCNF was applied was $17 \pm 5.3$ $\mathrm{nm}$. The corresponding histogram revealed that $65 \%$ of AgNPs were in the range of 7.5 to $16 \mathrm{~nm}$, and the remaining $30 \%$ and 5\% fractions showed a particle size distribution of 21 to $29 \mathrm{~nm}$ and 34 to $42 \mathrm{~nm}$, respectively. The average particle size of AgNPs obtained by using LCNF-5\% and LCNF-18\% were $12 \pm 3.3 \mathrm{~nm}$ regardless of the lignin content, and this size was smaller than that of HCNF. The corresponding histograms revealed that approximately 80 to $85 \%$ of AgNPs were distributed between 7.5 to $16 \mathrm{~nm}$, and the remaining 15 to $20 \%$ were distributed between 21 to $42 \mathrm{~nm}$. These results suggest that the lignin content controls the size of AgNPs.
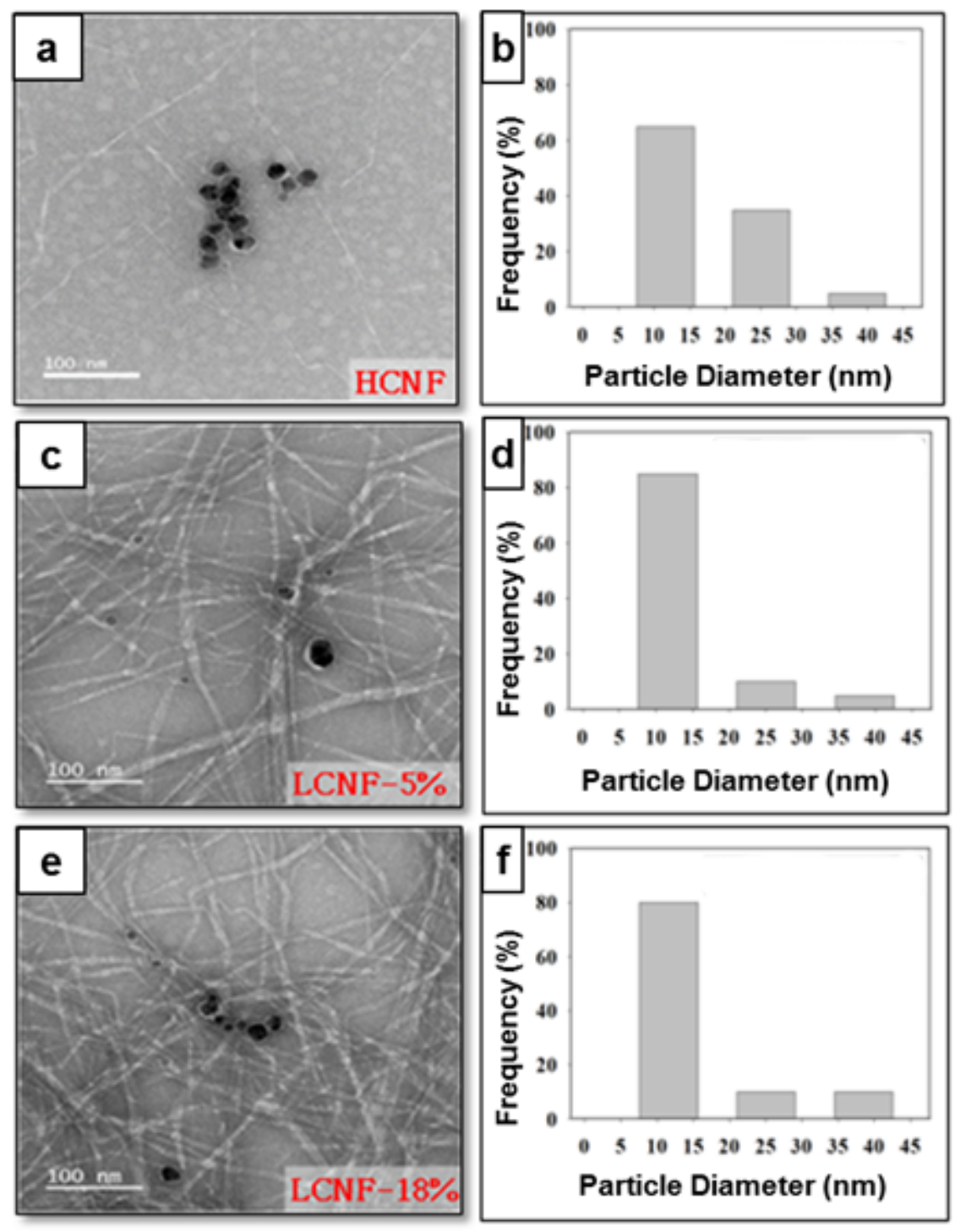

Fig. 4. High magnification TEM images of (a) AgNPs/HCNF, (c) AgNPs/LCNF-5\%, (e) AgNPs/LCNF-18\% and the corresponding particle size distribution of (b) AgNPs/HCNF, (d) AgNPs/LCNF-5\%, and (f) AgNPs/LCNF-18\% 


\section{XRD Analysis}

Figure 5 shows the powder XRD patterns of AgNPs synthesized using HCNF, LCNF-5\%, and LCNF-18\% as a reducing agent and stabilizer. Along with the characteristic peaks of $\mathrm{CNF}$, the figure clearly displays four other peaks at corresponding $2 \theta$ values of $38.3,44.3,64.4$, and 77.4. These peaks can be assigned to the 111, 200, 220, and 311 diffraction planes of the face centered cubic crystal structure of AgNPs, respectively (Indana et al. 2016). The XRD spectrum of AgNPs/LCNF was compared with the standard Ag crystalline metallic form (JCPDS-07-0783). The 111 diffraction peak was more intense than the other peaks, which clearly indicates that AgNPs are predominantly oriented along the 111 plane. The diffraction peaks are broad indicating that the crystallite size was very small (Rai et al. 2014). According to the obtained results, the cellulose crystalline structure of LCNF was unaltered after the formation of AgNPs. The characteristic diffraction peaks of AgNPs were more prominent in AgNPs/HCNF, while the same were very weak in AgNPs/LCNF-5\% and AgNPs/LCNF$18 \%$. This lower intensity of the peaks can be ascribed to the fewer amounts of AgNPs, which makes them distributed throughout the sample and difficult to be detected by the instrument (Dadigala et al. 2017). Furthermore the crystallite size of AgNPs (AgNPs/HCNF) was calculated to be $5.76 \mathrm{~nm}$ from (111) diffraction peak using the Debye-Scherrer equation (1).

$$
D=K \lambda / \beta \cos \theta
$$

In Eq. 1, the terms represent; $D$ crystallite size, $K$ constant $=0.9, \lambda$ wavelength of $\mathrm{X}$-ray source $(0.15405 \mathrm{~nm}), \beta$ full width at half maximum of (111) peak, and $\theta$ Bragg angle

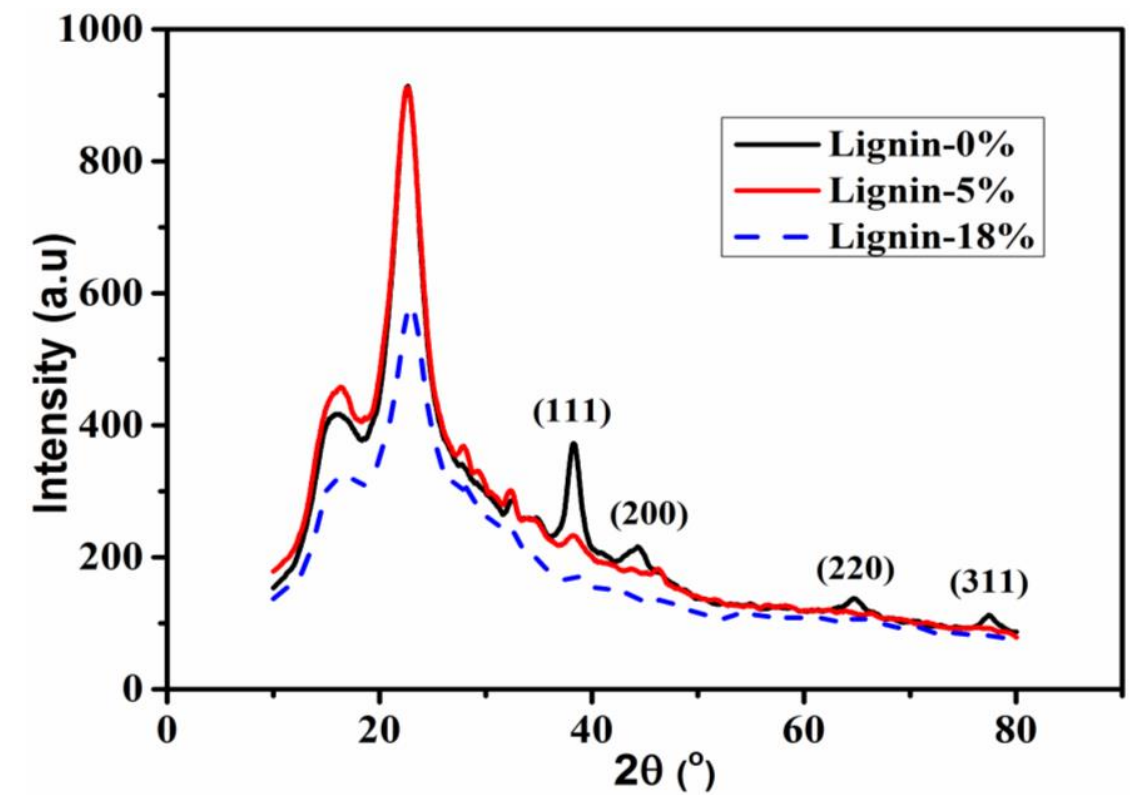

Fig. 5. XRD pattern of AgNPs/HCNF, AgNPs/LCNF-5\%, and AgNPs/LCNF-18\%

\section{FTIR Analysis}

The FTIR analysis of the freeze dried LCNF and AgNPs/LCNF composites were carried out to investigate the involvement of possible functional groups of the biopolymer in the reduction and stabilization of AgNPs. This analysis provides information about the structural changes of LCNF after autoclave. The comparative FTIR spectra of LCNF and 
AgNPs/LCNF are shown in Fig. 6. The FTIR spectrum of LCNF represents cellulose characteristic peaks at $3343.75,2891,1642,1316$, and $1030 \mathrm{~cm}^{-1}(\mathrm{OH}, \mathrm{C}-\mathrm{H}, \mathrm{C}=\mathrm{O}, \mathrm{OH}$, and $\mathrm{C}-\mathrm{C}$ ). The $1369 \mathrm{~cm}^{-1}$ peak was due to the presence of phenolic hydroxyl groups, and it was more intense in LCNF-18\%. The spectra of all LCNF show the vibrations characteristic for the guaiacyl unit $\left(1269 \mathrm{~cm}^{-1}, \mathrm{G}\right.$ ring and $\mathrm{C}=\mathrm{O}$ stretch, $\left.1140 \mathrm{~cm}^{-1}\right)$. The 1326 and $1323 \mathrm{~cm}^{-1}$ peaks can be assigned as syringyl (S) rings and syringyl and guaiacyl (G) condensed rings, which appeared in the spectra of lignin. A $1269 \mathrm{~cm}^{-1}$ peak is typical for $\mathrm{C}-\mathrm{O}$ and $\mathrm{C}=\mathrm{O}$ groups because of stretching in $\mathrm{G}$ rings. The strong vibration at 1212 to $1217 \mathrm{~cm}^{-1}$ is common for the spectra of LCNF. It can be associated with C-C, C-O, and $\mathrm{C}=\mathrm{O}$ stretching (Aracri et al. 2014). After the formation of AgNPs, a clear decrease in the intensities of the peaks corresponding to primary alcohol groups was observed, indicating their involvement in the formation of AgNPs.

The possible mechanism is anticipated for the reduction of Ag+ to AgNPs in a two-step process. In the first step, the negative zeta potential containing LCNF will interact with the positive silver ions to form an intermediate complex with hydroxyl groups present in LCNF. Furthermore, these hydroxyl groups will be oxidized to carbonyl or carboxyl forms by donating electron to Ag+ ions, which results in the reduction of $\mathrm{Ag}+$ ions and produce AgNPs. In the second step after the formation of AgNPs, the stabilization occurs through the strong association between AgNPs surface with oxygen atoms present in carbonyl and hydroxyl functional groups of the LCNF (Vinod et al. 2011).
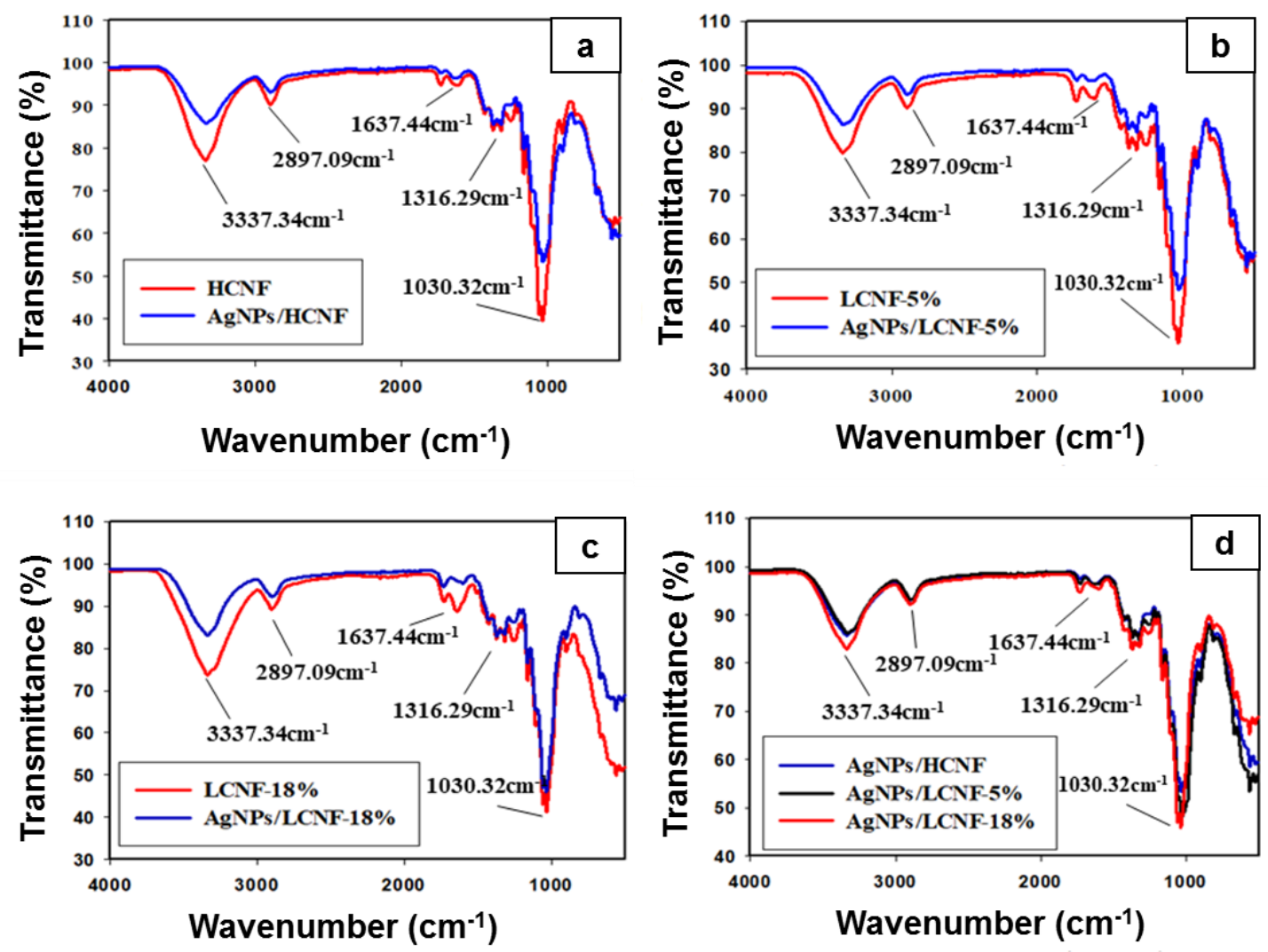

Fig. 6. FTIR spectra of (a) HCNF \& AgNPs/ HCNF, (b) LCNF-5\% and AgNPs/LCNF-5\%, (c) LCNF-18\% and AgNPs/LCNF-18\%, (d) Overlay spectrum of AgNPs/HCNF, AgNPs/LCNF 5\%, and $\mathrm{AgNPs} / \mathrm{LCNF}-18 \%$ 


\section{XPS Analysis}

The chemical states of silver exist in AgNPs, which were characterized by XPS and are shown in Fig. 7. Figure 7B is a detailed version of Fig. 7A. The XPS technique is an extremely influential tool to investigate the electronic and chemical structure at the molecule metal interface in the ligand capped nanoparticle samples. The silver contents on the surface of AgNPs prepared using HCNF, LCNF-5\%, and LCNF-18\% were $0.28 \%$, $0.16 \%$, and $0.09 \%$, respectively (Fig. 7B). Figure 7C is the high resolution XPS spectrum of AgNPs prepared using HCNF, LCNF-5\%, and LCNF-18\% which displayed 2 peaks at approximately $368 \mathrm{eV}$, and $374 \mathrm{eV}$ corresponding to the $\mathrm{Ag} 3 \mathrm{~d}_{5 / 2}$ and $\mathrm{Ag} 3 \mathrm{~d}_{3 / 2}$ components of elemental silver, respectively. This further confirms the successful reduction of $\mathrm{Ag}^{+}$to $\mathrm{Ag}^{0}$ by LCNF. Figure 7D is a detailed version of Fig. 7C. XPS results made it clear that the formation of AgNPs is greatly influenced by the amount of lignin present in the LCNF employed for the synthesis. The increased lignin content decreased the production yield of AgNPs.
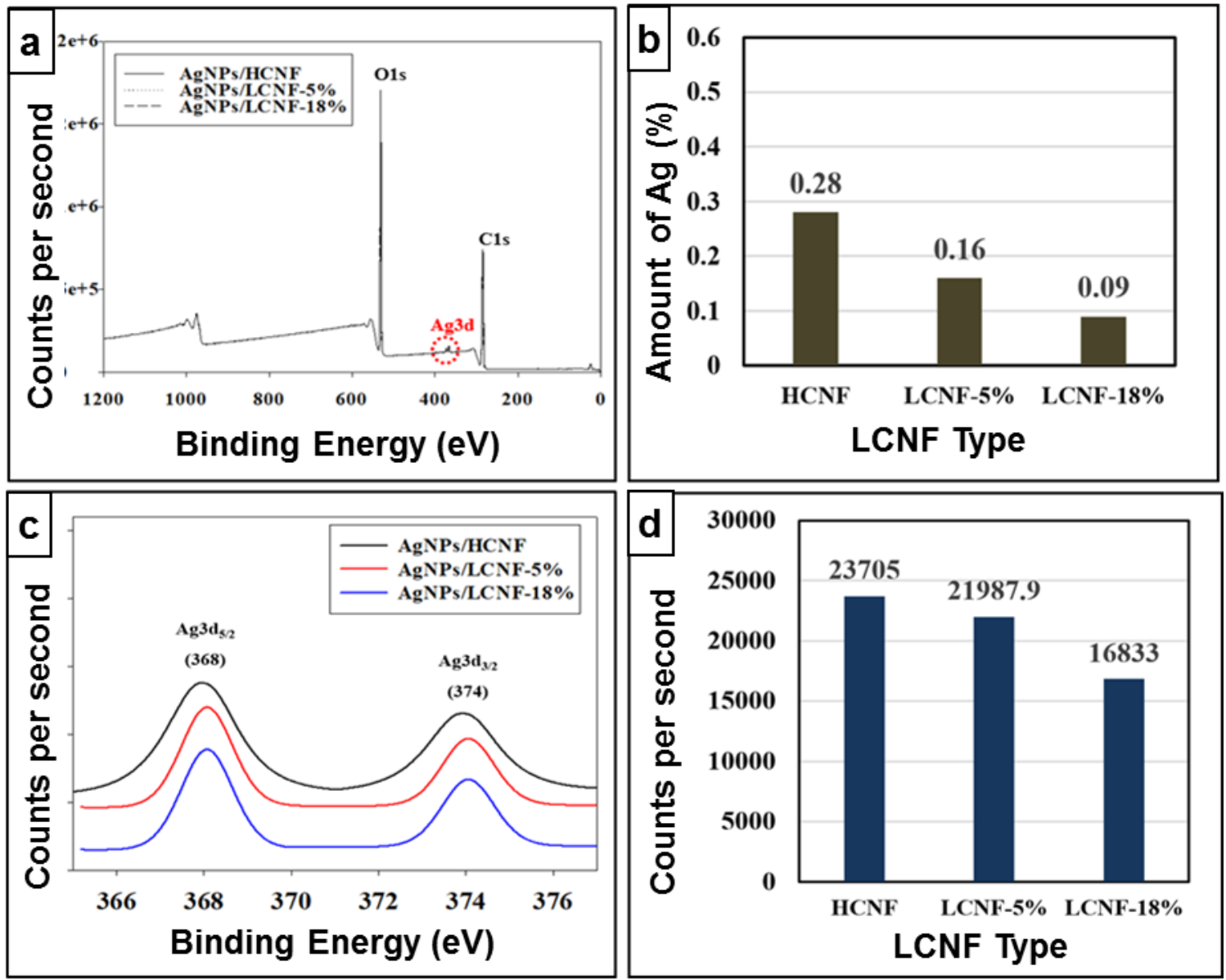

Fig. 7. (a) XPS spectra of AgNPs/HCNF, AgNPs/LCNF-5\%, and AgNPs/LCNF-18\%, (b) Silver \% of AgNPs/HCNF, AgNPs /LCNF-5\%, AgNPs/LCNF-18\%, (c) Ag3d spectra of AgNPs/HCNF, AgNPs /LCNF-5\%, and AgNPs /LCNF-18\%, (c) Counts on silver of AgNPs/HCNF, AgNPs /LCNF$5 \%$, and AgNPs /LCNF-18\% 


\section{CONCLUSIONS}

1. This study demonstrated a novel green method for producing AgNPs using lignocellulose nanofibrils (LCNF) as a reducing and stabilizing agent. A simple autoclave procedure was employed for the synthesis. The synthetic conditions such as concentrations of reactants and reaction time were optimized.

2. The effect of lignin content of LCNF on the formation of AgNPs was evaluated. For this purpose, three types of cellulose nanofibrils, i.e., HCNF (0\% lignin), LCNF-5 (5\% lignin), and LCNF-18 (18\% lignin), were employed to prepare AgNPs. Three types of AgNPs were thoroughly characterized.

3. The results of this study suggest that LCNF can be employed as a green source for the reduction and effective stabilization of AgNPs, but increased content of lignin had adverse effects on the yield of AgNPs. However, the presence of lignin greatly controlled the particle size. Therefore, LCNF with small amounts of lignin (5\%) is best for producing AgNPs.

\section{ACKNOWLEDGMENTS}

This study was funded by the Basic Science Research Program through the National Research Foundation of Korea (NRF) funded by the Ministry of Education Grant No. 2018R1A6A1A03025582.

\section{REFERENCES CITED}

Abou El-Nour, K. M. M., Eftaiha, A., Al-Warthan, A., and Ammar, R. A. A. (2010). "Synthesis and applications of silver nanoparticles," Arabian Journal of Chemistry 3(3), 135-140. DOI: 10.1016/j.arabjc.2010.04.008

Ahmed, S., Ahmad, M., Swami, B. L., and Ikram, S. (2016). "A review on plants extract mediated synthesis of silver nanoparticles for antimicrobial applications: A green expertise," Journal of Advanced Research 7(1), 17-28. DOI: 10.1016/j.jare.2015.02.007

Aracri, E., Díaz Blanco, C., and Tzanov, T. (2014). "An enzymatic approach to develop a lignin-based adhesive for wool floor coverings," Green Chemistry 16(5), 2597. DOI: $10.1039 / \mathrm{c} 4 \mathrm{gc} 00063 \mathrm{c}$

Cao, S., Ma, X., Lin, L., Huang, F., Huang, L., and Chen, L. (2014). "Morphological and chemical characterization of green bamboo (Dendrocalamopsis oldhami (Munro) Keng f.) for dissolving pulp production," BioResources 9(3). DOI: 10.15376/biores.9.3.4528-4539

Dadigala, R., Bandi, R., Gangapuram, B. R., and Guttena, V. (2017). "Carbon dots and Ag nanoparticles decorated g-C 3 N 4 nanosheets for enhanced organic pollutants degradation under sunlight irradiation," Journal of Photochemistry and Photobiology A: Chemistry 342, 42-52. DOI: 10.1016/j.jphotochem.2017.03.032

Dong, H., Snyder, J. F., Tran, D. T., and Leadore, J. L. (2013). "Hydrogel, aerogel and film of cellulose nanofibrils functionalized with silver nanoparticles," Carbohydrate Polymers 95(2), 760-767. DOI: 10.1016/j.carbpol.2013.03.041 
Gopiraman, M., Deng, D., Saravanamoorthy, S., Chung, I. M., and Kim, I. S. (2018). "Gold, silver and nickel nanoparticle anchored cellulose nanofiber composites as highly active catalysts for the rapid and selective reduction of nitrophenols in water," RSC Advances 8(6), 3014-3023. DOI: 10.1039/c7ra10489h

Habibipour, R., Moradi-Haghgou, L., and Farmany, A. (2019). "Green synthesis of AgNPs@PPE and its Pseudomonas aeruginosa biofilm formation activity compared to pomegranate peel extract," International Journal of Nanomedicine 14, 6891-6899. DOI: $10.2147 /$ IJN.S209912

Hyman, D., Sluiter, A., Crocker, D., Johnson, D., Sluiter, J., Black, S., and Scarlata, C. (2008). Determination of Acid Soluble Lignin Concentration Curve by UV-Vis Spectroscopy (NREL/TP-510-42617), National Renewable Energy Laboratory, Golden, CO, USA.

Indana, M. K., Gangapuram, B. R., Dadigala, R., Bandi, R., and Guttena, V. (2016). “A novel green synthesis and characterization of silver nanoparticles using gum tragacanth and evaluation of their potential catalytic reduction activities with methylene blue and Congo red dyes," Journal of Analytical Science and Technology 7(1), 1-9. DOI: 10.1186/s40543-016-0098-1

Khan, I., Saeed, K., and Khan, I. (2019). "Nanoparticles: Properties, applications and toxicities," Arabian Journal of Chemistry 12(7), 908-931. DOI: 10.1016/j.arabjc.2017.05.011

Lee, J. S., Lytton-Jean, A. K. R., Hurst, S. J., and Mirkin, C. A. (2007). "Silver nanoparticle-oligonucleotide conjugates based on DNA with triple cyclic disulfide moieties," Nano Letters 7(7), 2112-2115. DOI: 10.1021/nl071108g

Li, X., and Lenhart, J. J. (2012). "Aggregation and dissolution of silver nanoparticles in natural surface water," Environmental Science and Technology 46(10), 5378-5386. DOI: 10.1021/es204531y

Madhusudhan, A., Reddy, G. B., and Krishana, I. M. (2019). "Green synthesis of gold nanoparticles by using natural gums," Nanomaterials and Plant Potential 111-134. DOI: 10.1007/978-3-030-05569-1_4

Narayanan, K. B., and Sakthivel, N. (2010). "Biological synthesis of metal nanoparticles by microbes," Advances in Colloid and Interface Science 156(1-2), 1-13. DOI: 10.1016/j.cis.2010.02.001

Natsuki, J. (2015). "A review of silver nanoparticles: Synthesis methods, properties and applications," International Journal of Materials Science and Applications 4(5), 325. DOI: $10.11648 /$ j.ijmsa.20150405.17

Ovais, M., Khalil, A. T., Raza, A., Khan, M. A., Ahmad, I., Islam, N. U., Saravanan, M., Ubaid, M. F., Ali, M., and Shinwari, Z. K. (2016). "Green synthesis of silver nanoparticles via plant extracts: beginning a new era in cancer theranostics," Nanomedicine 11(23), 3157-3177. DOI: 10.2217/nnm-2016-0279

Oves, M., Aslam, M., Rauf, M. A., Qayyum, S., Qari, H. A., Khan, M. S., Alam, M. Z., Tabrez, S., Pugazhendhi, A., and Ismail, I. M. I. (2018). "Antimicrobial and anticancer activities of silver nanoparticles synthesized from the root hair extract of Phoenix dactylifera," Materials Science and Engineering: C 89, 429-443. DOI: 10.1016/j.msec.2018.03.035

Park, C. W., Han, S. Y., Choi, S. K., and Lee, S. H. (2017a). "Preparation and properties of holocellulose nanofibrils with different hemicellulose content," BioResources 12(3), 6298-6308. DOI: 10.15376/biores.12.3.6298-6308

Park, C. W., Han, S. Y., Namgung, H. W., Seo, P. N., Lee, S. Y., and Lee, S. H. (2017b). 
"Preparation and characterization of cellulose nanofibrils with varying chemical compositions," BioResources 12(3), 5031-5044. DOI: 10.15376/biores.12.3.50315044

Prabhu, S., and Poulose, E. K. (2012). "Silver nanoparticles: mechanism of antimicrobial action, synthesis, medical applications, and toxicity effects," International Nano Letters, 2(1), 32. DOI: 10.1186/2228-5326-2-32

Pugazhendhi, A., Edison, T. N. J. I., Karuppusamy, I., and Kathirvel, B. (2018). "Inorganic nanoparticles: A potential cancer therapy for human welfare," International Journal of Pharmaceutics, 539(1-2), 104-111. DOI:

10.1016/j.ijpharm.2018.01.034

Rafique, M., Sadaf, I., Rafique, M. S., and Tahir, M. B. (2017). "A review on green synthesis of silver nanoparticles and their applications," Artificial Cells, Nanomedicine and Biotechnology 45(7), 1272-1291. DOI: 10.1080/21691401.2016.1241792

Rai, M., Kon, K., Ingle, A., Duran, N., Galdiero, S., and Galdiero, M. (2014). "Broadspectrum bioactivities of silver nanoparticles: The emerging trends and future prospects," Applied Microbiology and Biotechnology 98(5), 1951-1961. DOI: 10.1007/s00253-013-5473-x

Rajoriya, P., Misra, P., Singh, V. K., Shukla, P. K., and Ramteke, P. W. (2017). "Green synthesis of silver nanoparticles," Biotech Today: An International Journal of Biological Sciences 7(1), 7. DOI: 10.5958/2322-0996.2017.00001.1

Saratale, G. D., Saratale, R. G., Benelli, G., Kumar, G., Pugazhendhi, A., Kim, D.-S., and Shin, H.-S. (2017). "Anti-diabetic Potential of silver nanoparticles synthesized with argyreia nervosa leaf extract high synergistic antibacterial activity with standard antibiotics against foodborne bacteria," Journal of Cluster Science 28(3), 1709-1727. DOI: $10.1007 / \mathrm{s} 10876-017-1179-\mathrm{z}$

Saravanan, M., Barik, S. K., MubarakAli, D., Prakash, P., and Pugazhendhi, A. (2018). "Synthesis of silver nanoparticles from Bacillus brevis (NCIM 2533) and their antibacterial activity against pathogenic bacteria," Microbial Pathogenesis 116, 221226. DOI: 10.1016/j.micpath.2018.01.038

Suman, T. Y., Elumalai, D., Kaleena, P. K., and Rajasree, S. R. R. (2013). "GC-MS analysis of bioactive components and synthesis of silver nanoparticle using Ammannia baccifera aerial extract and its larvicidal activity against malaria and filariasis vectors," Industrial Crops and Products 47, 239-245. DOI:

10.1016/j.indcrop.2013.03.010

Van Rie, J., and Thielemans, W. (2017). "Cellulose-gold nanoparticle hybrid materials," Nanoscale 9(25), 8525-8554. DOI: $10.1039 / \mathrm{c} 7 \mathrm{nr} 00400 \mathrm{a}$

TAPPI T204 cm-97 (2002). "Solvent extractives of wood pulp," TAPPI Press, Atlanta, GA.

TAPPI T222 om-11 (2006). “Acid-insoluble lignin in wood and pulp," TAPPI Press, Atlanta, GA.

Vinod, V. T. P., Saravanan, P., Sreedhar, B., Devi, D. K., and Sashidhar, R. B. (2011). "A facile synthesis and characterization of Ag, Au and Pt nanoparticles using a natural hydrocolloid gum kondagogu (Cochlospermum gossypium)," Colloids and Surfaces B: Biointerfaces 83(2), 291-298. DOI: 10.1016/j.colsurfb.2010.11.035

Wang, M. S., Jiang, F., Hsieh, Y. Lo, and Nitin, N. (2014). "Cellulose nanofibrils improve dispersibility and stability of silver nanoparticles and induce production of bacterial extracellular polysaccharides," Journal of Materials Chemistry B 2(37), 
6226-6235. DOI: 10.1039/c4tb00630e

Xu, Y., Zuo, L., Qian, X., and Wang, J. (2016). "Preparation and characterization of cellulose-silver nanocomposites by in situ reduction with alkalis as activation reagent," BioResources 11(1), 2797-2808. DOI: 10.15376/biores.11.1.2797-2808

Article submitted: October 3, 2019; Peer review completed: December 21, 2019; Revised version received and accepted: January 22, 2020; Published: February 3, 2020.

DOI: 10.15376/biores.15.2.2119-2132 